\title{
Tecnologias e interação em famílias com adolescente: comunicação
}

\section{entre mães e filhos}

\section{Technologies and interaction in families with adolescents: Communication between mothers and offspring}

\author{
Rita Martinho, Heldemerina Pires \\ Universidade de Évora;
}

\begin{abstract}
Resumo
$\mathrm{Na}$ adolescência, a interação entre adolescentes e pais assume uma nova dimensão. A busca pela autonomia, a construção da identidade e os pares adquirem grande importância. Acresce que o desenvolvimento e utilização de tecnologias, sendo estas necessárias às rotinas das pessoas, tornaram-se suscetíveis de influenciar a interação familiar, particularmente, a comunicação. Mediante estudo qualitativo e realização de entrevista semiestruturada analisou-se a influência das tecnologias na comunicação em famílias com adolescentes. Participaram no estudo 13 mães e respetivos filhos/as. Dos dados sujeitos a análise de conteúdo concluiu-se que as tecnologias influenciam a comunicação entre mães e adolescentes, facilitando-a ou dificultando-a.

Palavras-chave: interação familiar, adolescência, comunicação, tecnologia.
\end{abstract}

\begin{abstract}
In adolescence, the interaction between adolescents and parents assumes a new dimension. The pursuit of autonomy, the self-identity development and the peers acquire a great importance. Furthermore, the increasingly development and widespread use of technologies, which are necessary to people's dailylife routine, became susceptible to influence family interaction, particularly, communication. Through a qualitative study and a semistructured interview, the influence of technologies in family communication in families with adolescents was analyzed. Thirteen mothers and their offspring participated in the study. From the data subjected to content analysis, it was concluded that technologies do influence communication between mothers and adolescents, whether facilitating or hindering it.

Keywords: family interaction, adolescence, communication, technology.
\end{abstract}

A adolescência é perspetivada como uma etapa do desenvolvimento onde ocorrem transformações em vários domínios (e.g., biológico psicológico, emocional, cognitivo, social) (Fonseca, 2012). A construção da identidade e a concretização da autonomia são duas tarefas fundamentais para os/as adolescentes e
Neuenschwander (2002) considera que estas permitemlhes definirem a sua identidade e o modo como se percecionam e iniciam o processo de diferenciação e autonomização face aos pais (Beja \& Franco, 2013). Deste modo, os/as adolescentes vão adquirindo uma crescente autonomia que lhes favorece o contacto no exterior do sistema familiar.

Durante a adolescência, para além das fronteiras do sistema familiar sofrerem um aumento na sua flexibilização face ao exterior e verifica-se que na família as relações entre pais e filhos/as sofrem transformações, nomeadamente: a modificação gradual da relação vertical entre pais e filho/a para uma relação cada vez mais simétrica Mota e Rocha (2012); De Goede, Branje e Meeus (2009), conduzindo a uma redefinição do posicionamento do sistema parental (Wagner, Falcke, Silveira \& Mosmann, 2002). Observase também que pais e filhos/as passam menos tempo juntos e realizam um menor número de atividades e de tarefas em conjunto (Alarcão, 2006). Neste sentido, a interação entre pais e adolescente pode caracterizar-se por uma pobre comunicação e uma expressão emocional negativa sendo resultado da importância que o grupo de pares adquire para o/a adolescente (Rodríguez \& López 1999). Esta situação leva a que os pais se sintam como figuras de vinculação de "reserva" ao serem menos solicitados, sentindo-se menos úteis, menos obedecidos e podendo perder alguma autoridade (Alarcão, 2006). $\mathrm{Na}$ realidade, o grupo de pares revela-se um grupo exterior à família altamente significativo para o/a adolescente por ser concetualizado como um suporte instrumental e emocional que proporciona a oportunidade de construir e manter as amizades (Kirchler, Palmonari \& Pombeni, 1993).

Para além do grupo de pares, as tecnologias revelaram-se um outro tipo de influências externas que podem constituir-se como agentes socializadores de referência capazes de contrastar, complementar, potenciar ou anular a influência de agentes socializadores de pertença como a família, o grupo de 
pares ou a escola (Abreu, 2012). Não obstante, as tecnologias são naturalmente externas à família, mas atualmente estão inseridas no contexto familiar, verificando-se uma presença acentuada de dispositivos tecnológicos que se caracterizam por se terem tornado indispensáveis e intrínsecos à rotina contemporânea (Church, Weight, Berry \& MacDonald, 2010).

Segundo Prados, Vicent e Esteban (2014), a introdução e presença das tecnologias no contexto familiar tem sido cada vez mais significativa conduzindo a um impacto na interação e comunicação entre os indivíduos da família. Por um lado, encontramse na literatura estudos que corroboram a hipótese das tecnologias facilitarem a interação social e por outro, existem estudos que mostram que as tecnologias podem levar ao isolamento.

Enquanto facilitadoras da interação social, as tecnologias têm sido utilizadas em atividades independentes e, simultaneamente são consideradas como instrumentos de socialização e comunicação (Villegas, 2013). Neste sentido, Church, Weight, Berry e MacDonald (2010) consideram que estas servem as necessidades pessoais, nomeadamente, (i) originam relações funcionais e envolvem o mundo social, ajudando na prestação de atividades de lazer comum, reunindo as gerações e simultaneamente estimular e permitir a comunicação familiar; (ii) ajudam na regulação do tempo e do espaço familiares, incluindo as tecnologias para a definição de atividades apropriadas ou horários (e.g., trabalhos de casa, hora de dormir); e (iii) podem promover oportunidades para o desenvolvimento de atividades partilhadas.

McGrath (2012) e Villegas (2013) referem que as tecnologias podem, possibilitar a interação social, mas, simultaneamente podem conduzir os membros das famílias em atividades solitárias (Daly, 1996 como citado em Mesch, 2006; McGrath, 2012).

Além das vantagens supramencionadas, as tecnologias podem ser prejudiciais na interação dos indivíduos no contexto familiar. A este respeito Rideout, Foehr e Roberts (2010) e Saxbe, Graesch e Alvik (2013) referem que o uso individualizado da televisão e do computador podem contribuir para o isolamento de crianças e adolescentes por estes preferirem utilizá-los sozinhos no quarto. Hameededdin (2010) destaca que o telemóvel encoraja os indivíduos a interagir com outros, porém este uso não é presencial, diminuindo o contacto próximo. No caso dos videojogos, Redmont (2010) argumenta que as horas passadas a jogar, podem ser associadas a uma diminuição na comunicação dos/as adolescentes com os irmãos.

A interação social e a comunicação na família, considerando os aspetos anteriormente referidos, podem ser afetadas pelas tecnologias. Por um lado, estas possibilitam momentos de união, de comunicação, de partilha de experiências e de aprendizagem entre a família, por outro lado podem contribuir para o isolamento dos indivíduos pelo caráter individual de algumas atividades, reduzindo a interação com os demais ou mesmo originar conflitos intrafamiliares.
Quanto ao presente estudo, assumida a importância da comunicação no contexto familiar, existe a preocupação de compreender de que modo as tecnologias influenciam a comunicação em famílias com filhos adolescentes, no seu quotidiano, analisando mais precisamente: i) a perceção da relação entre o tempo utilizado pelo/a adolescente com tecnologias e a sua comunicação com a família; ii) a perceção de como seria a comunicação entre o adolescente e a família se não existissem tecnologias.

\section{Método}

\section{Participantes}

Este estudo foi desenvolvido com a participação de $(\mathrm{N}=13)$ mães com idades compreendidas entre os $41 \mathrm{e}$ os 54 anos e respetivos filhos/as adolescentes $(\mathrm{N}=14)$ de ambos os sexos, ( 7 do sexo feminino e 7 do sexo masculino) com idades compreendidas entre os 14 e os 18 anos. A maioria das mães $(\mathrm{N}=9)$ e dos/as adolescentes $(\mathrm{N}=9)$ admite ter algum conhecimento sobre tecnologias.

\section{Instrumentos}

Para a recolha de dados, do presente estudo, foi utilizada uma entrevista semiestruturada assente num guião organizado a partir de objetivos propostos à priori, os quais serviram de base à formulação das questões.

\section{Procedimentos}

Foram definidos os seguintes critérios de inclusão no estudo: ser pai ou mãe de adolescente com idade compreendida entre os 14 e os 18 anos; possibilidade de entrevistar o par mãe/filho/a e/ou pai/filho/a. Devido a indisponibilidade dos pais para participar no estudo, apenas foram entrevistadas as mães e respetivos filhos/as.

As entrevistas foram realizadas após a obtenção das autorizações e consentimentos informados tendo sido gravadas e posteriormente transcritas e codificadas de modo a garantir o anonimato dos entrevistados. Para a análise dos resultados, foi utilizada a técnica de análise de conteúdo. Por se tratar de um processo indutivo de análise dos dados, as categorias foram criadas à posteriori (Moraes, 1999). Foram definidos dois tipos de unidade (UR e UC). As unidades de registo (UR), são palavras e expressões chave do discurso dos/as participantes que permitem organizar as categorias dentro de um determinado objetivo em análise; e as unidades de contagem (UC), dizem respeito ao número de vezes que cada entrevistado/a faz referência a determinada experiência.

\section{Resultados}

Dos dados em análise relativos ao primeiro objetivo: perceber a relação entre o tempo utilizado pelo adolescente com tecnologias e a sua comunicação com a familia, emergiram três categorias: Categoria 1 Relação Positiva, (a utilização das tecnologias facilita a comunicação e interação familiares); Categoria 2 Relação Negativa (as tecnologias podem prejudicar a 
comunicação e interação familiares) e Categoria 3 Relação Neutra (as tecnologias parecem não ter influência sobre a comunicação e a interação familiares) e respetivas subcategorias.

Tabela 1

Categoria 1: Relação positiva (verbalizações de mães)

\begin{tabular}{lcc}
\hline Subcategorias & UR & UC \\
\hline Facilidade de Contacto & 2 & 2 \\
Aprendizagem de Conteúdos & 3 & 3 \\
\hline
\end{tabular}

A Tabela 1 apresenta as subcategorias da Categoria 1 - Relação Positiva. Esta categoria emergiu apenas das verbalizações das mães. Estas apresentam uma perceção positiva a respeito da relação entre tempo utilizado pelo/a adolescente com tecnologias e a sua comunicação com a família. Como se pode ver na Tabela 1, destas verbalizações destaca-se que a utilização das tecnologias facilita o contacto e a comunicação entre os elementos da família "É bom porque ela está contactável" M9 por um lado e por outro, dá a possibilidade de aprendizagem de conteúdos "Influencia às vezes no bom sentido porque ele também aprende e ajuda-me" M10.

Tabela 2

Categoria 2: Relação negativa (verbalizações de mães e de adolescentes)

\begin{tabular}{lcccc}
\hline & \multicolumn{2}{c}{ Mães } & \multicolumn{2}{c}{ Adolescentes } \\
\hline Subcategorias & UR & UC & UR & UC \\
\hline $\begin{array}{l}\text { Diminuição da } \\
\text { Comunicação }\end{array}$ & 8 & 8 & 8 & 8 \\
$\begin{array}{l}\text { Isolamento do/a } \\
\text { Adolescente }\end{array}$ & 1 & 1 & - & - \\
\hline
\end{tabular}

A Tabela 2 apresenta as subcategorias da Categoria 2 - Relação Negativa. Esta categoria emergiu das verbalizações das mães e dos/as adolescentes que apresentam uma perceção negativa sobre a relação entre o tempo utilizado pelo/a adolescente com tecnologias e a sua comunicação com a família. Como se verifica na Tabela 2, destaca-se que $(\mathrm{N}=3)$ mães e $(\mathrm{N}=7)$ adolescentes verbalizam que a utilização de tecnologias conduz à diminuição da comunicação "Se ele se calhar não passasse tanto tempo no computador, estava aqui mais presente a conversar com a mãe dele" M4; “Às vezes, em vez de estar no telefone, podia estar mais tempo com eles [pai e mãe]" A9. A perceção negativa sobre a relação entre o tempo utilizado pelo/a adolescente com tecnologias e a sua comunicação com a família também pode estar associada ao isolamento do/a adolescente $(\mathrm{N}=1)$ mãe "Porque ele já era uma criança fechada sem a tecnologia, agora então" M13.

Tabela 3

Categoria 3: Relação neutra (verbalizações de mães e de adolescentes)

\begin{tabular}{lcccc}
\hline & \multicolumn{2}{c}{ Mães } & \multicolumn{2}{c}{ Adolescentes } \\
\hline Categoria & UR & UC & UR & UC \\
\hline Relação Neutra & 6 & 6 & 8 & 8 \\
\hline
\end{tabular}

Na Tabela 3 apresenta-se apenas o resultado das verbalizações referentes à Categoria 3 - Relação Neutra, visto que não emergiram subcategorias. Esta categoria surgiu das verbalizações das mães e dos/as adolescentes que apresentam uma perceção neutra sobre a relação entre o tempo utilizado pelo/a adolescente com tecnologias e a sua comunicação com a família. Como se pode ver na Tabela 3 , pelas verbalizações de $(\mathrm{N}=6)$ mães e $(\mathrm{N}=8)$ adolescentes parece haver uma perceção de que o tempo passado com tecnologias não tem influência na comunicação do adolescente com a família "Eu acho que não influencia muito (...) acabamos por ter tempo disponível para falar os dois, portanto não é as tecnologias que nos barram a ligação ou a comunicação entre os dois" M6; "No meu caso acho que não influencia muito porque quando não ligava muito às tecnologias também não falava muito com eles [pai e mãe]" A13.

Da análise dos dados relativos ao segundo objetivo: perceber como seria a comunicação entre o/a adolescente e a família se não existissem tecnologias, emergiram duas categorias: 1 - Comunicação Igual, de acordo com as verbalizações dos/as participantes, sem as tecnologias a comunicação entre o/a adolescente e seus pais manter-se-ia igual; 2 - Comunicação Diferente, sem as tecnologias a comunicação entre o/a adolescente e seus pais seria diferente; e respetivas subcategorias.

Tabela 4

Categoria 1: Comunicação igual (verbalizações de Mães e de Adolescentes)

\begin{tabular}{lcccc}
\hline & \multicolumn{2}{c}{ Mães } & \multicolumn{2}{c}{ Adolescentes } \\
\hline Subcategorias & UR & UC & UR & UC \\
\hline Comunicação Igual & 4 & 4 & 8 & 8 \\
$\begin{array}{l}\text { Modificação de } \\
\text { Hábitos }\end{array}$ & 1 & 1 & - & - \\
\hline
\end{tabular}

A Tabela 4 apresenta as subcategorias da Categoria 1 - Comunicação igual. Esta categoria emergiu das verbalizações das mães e dos/as adolescentes que consideram que sem as tecnologias a comunicação do/a adolescente com os pais manter-se-ia igual, não sofrendo alterações. Como é possível verificar na Tabela 4, destas verbalizações destaca-se que $(\mathrm{N}=4)$ mães e $(\mathrm{N}=8)$ adolescentes têm a perceção de que a comunicação sem tecnologias seria igual: "Entre mim e ela não seria muito diferente (...) se não estivesse a mexer no computador ou no Tablet, ela estaria a fazer desenhos, a escrever, a ler" M8; "Acho que seria praticamente igual, não sei, acho que sim, seria igual" A14. A subcategoria Modificação de Hábitos surge da verbalização de uma mãe que considera que nesse contexto a comunicação seria igual e apenas haveria uma modificação dos hábitos já adquiridos, "Devo reconhecer que aquilo que mudaria seria mais ao nível dos hábitos do que propriamente do grau de comunicação (...) mas eu não acho que a comunicação fosse melhor por causa disso" M7. 
Tabela 5

Categoria 2: Comunicação diferente (verbalizações de mães e de adolescentes)

\begin{tabular}{|c|c|c|c|c|}
\hline & \multicolumn{2}{|c|}{ Mães } & \multicolumn{2}{|c|}{ Adolescentes } \\
\hline Subcategorias & UR & $\mathbf{U C}$ & UR & $\mathbf{U C}$ \\
\hline $\begin{array}{l}\text { Melhoria da } \\
\text { Comunicação }\end{array}$ & 8 & 8 & 8 & 8 \\
\hline $\begin{array}{l}\text { Diminuição da } \\
\text { Comunicação }\end{array}$ & 1 & 1 & - & - \\
\hline $\begin{array}{l}\text { Reorganização do } \\
\text { Planeamento das } \\
\text { Rotinas }\end{array}$ & - & - & 3 & 3 \\
\hline
\end{tabular}

A Tabela 5 apresenta as subcategorias da Categoria 2 - Comunicação Diferente. Esta categoria emergiu das verbalizações das mães e dos/as adolescentes que consideram que sem as tecnologias a comunicação do/a adolescente com os pais seria diferente. Como é possível verificar na Tabela 5, destaca-se que sem tecnologias a comunicação seria melhor $(\mathrm{N}=8)$ mães e $(\mathrm{N}=8)$ adolescentes "Talvez a gente conversasse mais ou fizéssemos mais outro tipo de jogos" M4; "Era boa porque assim, se não houvesse tecnologia, podia falar com os meus pais sempre, estar sempre a falar e dar passeios e isso tudo" A3. A subcategoria: Diminuição da Comunicação/Contacto, surgiu da verbalização de uma mãe que considera que haveria uma redução da comunicação pela impossibilidade de contactar o/a filho/a durante o dia, "Se não houvesse telemóvel aquilo era uma coisa muito má, uma pessoa que entra para o trabalho logo cedo e só via o filho à tardinha, era muito complicado mesmo" M11. A subcategoria: Reorganização do Planeamento das Rotinas, surge da perceção de $(\mathrm{N}=3)$ adolescentes para quem a comunicação seria diferente por conduzir à reorganização e planeamento das rotinas "Em termos do meu dia teria que ser melhor planeado porque depois não ia fazer chamadas (...) e enviar mensagens a avisar que vou para ali. Tinha que planear logo tudo" A8.

\section{Discussão}

Esta investigação teve como objetivo analisar de que modo as tecnologias influenciam a comunicação em famílias com filhos/as adolescentes, no seu quotidiano, analisando mais precisamente: (i) a perceção da relação entre o tempo utilizado pelo/a adolescente com tecnologias e a sua comunicação com a família; (ii) a perceção de como seria a comunicação entre o adolescente e a família se não existissem tecnologias.

Conhecer a perceção da relação entre o tempo utilizado pelo adolescente com tecnologias e a comunicação com a família: Neste primeiro objetivo, dos resultados obtidos emergem três categorias (relação positiva, negativa e neutra). De acordo com as perceções dos/as entrevistados/as, é notório que a presença de tecnologias em casa tem um impacto significativo na forma como a família comunica e interage. De acordo com os/as participantes da investigação, pela perceção das mães $(\mathrm{N}=2)$ as tecnologias parecem facilitar a comunicação e interação familiares focando essencialmente a facilidade de contacto entre as mães e os/as filhos/as e na aprendizagem de conteúdos, em que os/as mais novos podem ensinar e auxiliar os pais em tarefas que envolvam tecnologias, por um lado (Lanigan, Bold \& Chenoweth, 2009; Saxbe, Graesh \& Alvik, 2011; Prados, Vicent \& Esteban, 2014). Por outro lado, de acordo com a perceção dos/as adolescentes $(\mathrm{N}=7)$ e mães $(\mathrm{N}=3)$, as tecnologias podem prejudicar a comunicação e interação familiares por retirar tempo que estes/as poderiam dedicar a interagir com os pais diminuindo deste modo a comunicação familiar (Quintas, 1998; Subrahmanyam, Kraut, Greenfield \& Gross, 2000; Morisson \& Krugman, 2001; Redmont, 2010). O resultado sugere que os/as adolescentes parecem estar conscientes do tempo excessivo despendido com tecnologias e consequente efeito negativo que, o modo como utilizam esse tempo, tem na comunicação e interação com a família, em particular os progenitores. Considerando ainda a relação negativa entre o tempo despendido com tecnologias e a comunicação do/a adolescente com os pais, os resultados observados evidenciam o isolamento dos/as adolescentes. A perceção das mães relativamente ao isolamento a que os/as adolescentes podem estar sujeitos/as pode sustentar-se pelo conceito da "Cultura de Quarto" referido por Bovill e Livingstone (2001). Caracterizando este fenómeno, as autoras, referem que as crianças e adolescentes passam uma parte considerável do "tempo de lazer em casa com as tecnologias em vez de passarem o seu tempo com a família ou no espaço familiar" (p.3). De facto, uma grande parte dos quartos das crianças e dos adolescentes europeus tornaram-se num espaço de lazer. A presença deste fenómeno, de facto, reforça a tendência ao isolamento do/a adolescente e à diminuição do convívio com a família. Contudo, através dos dados obtidos, também é possível observar que segundo a perceção de $(\mathrm{N}=6)$ mães e $(\mathrm{N}=8)$ adolescentes, o tempo despendido com tecnologias parece não ter qualquer impacto na comunicação entre o/a adolescente e seus pais, notandose que as tecnologias não influenciam positiva ou negativamente a comunicação e a interação com a família.

Conhecer a perceção de como seria a comunicação entre adolescente e família se não existissem tecnologias: Neste segundo objetivo, analisando as perceções dos/as participantes, dos resultados emergem duas categorias: comunicação igual (a comunicação não sofreria alterações face à inexistência das tecnologias) e comunicação diferente (a comunicação sofreria alterações face à inexistência de tecnologias). As mães consideram que sem tecnologias, a comunicação com o/a adolescente manter-se-ia igual, não se proporcionando uma maior comunicação. Esta perceção pode justificar-se por existir no período da adolescência entre os pais e o/a adolescente uma comunicação pobre e uma expressão emocional negativa (Rodríguez \& López, 1999). Daí que as mães percecionem que, mesmo sem tecnologias, a comunicação com os/as filhos/as seria igual, sugerindo que pouca comunicação entre os pais/mães e filhos/as adolescentes é natural 
neste período. Existe concordância de respostas dos/as adolescentes (tabela 4 e 3 ) onde é evidente que as tecnologias não influenciam a comunicação e a interação com o pai e com a mãe.

Caso não existissem tecnologias, a segunda categoria (comunicação diferente) surgida das perceções das mães $(\mathrm{N}=8)$ revela que a comunicação com o/a filho/a poderia melhorar, prevendo-se um aumento dos momentos de comunicação e interação familiar. Este resultado está em concordância com os resultados apresentados na Tabela 2 (relação negativa entre o tempo despendido com tecnologias e a comunicação e interação com a família) onde as mães referem que as tecnologias retiram tempo aos/às adolescentes que poderiam despender com a família.

Os/as adolescentes, também referiram que sem as tecnologias a comunicação com os pais seria diferente no sentido de uma melhoria. Relacionando-se estes resultados com os resultados da Tabela 2, é pertinente referir que os mesmos coincidem. $\mathrm{O}$ resultado sugere que, os/as adolescentes parecem ter consciência de que as tecnologias retiram tempo que poderia ser aproveitado para comunicar e interagir com a família, particularmente com os pais. O resultado leva-nos a inferir-se que, apesar de gostarem de estar com tecnologias, os/as adolescentes entendem que a comunicação com os pais pode ser importante. Os/as adolescentes participantes no estudo ainda percecionam que, caso não existissem tecnologias, a sua rotina diária teria que ser reorganizada, pois sem o acesso a tecnologias, não poderiam contactar facilmente os pais ou outras pessoas, sendo necessário um planeamento prévio do dia. Este dado pode sugerir que as tecnologias, ao estarem incluídas na família, têm um papel importante na organização das suas rotinas (Spigel, 1992 como citado em Church, 2010).

Desta investigação, pode-se concluir que as tecnologias influenciam a comunicação e interação na família. No que respeita ao primeiro objetivo da investigação conclui-se que o tempo que os adolescentes despendem com tecnologias pode influenciar e afetar a comunicação e a interação destes/as com a família, nomeadamente: (i) Facilitando$a$ (Lanigan, Bold \& Chenoweth, 2009; Saxbe, Graesh \& Alvik, 2011; Prados, Vicent \& Esteban, 2014) (ii) Prejudicando-a (Quintas, 1998; Subrahmanyam, Kraut, Greenfield \& Gross, 2000; Morisson \& Krugman, 2001; Redmont, 2010); Ou ainda (iii) não tendo qualquer tipo de influência (Mieczakowskim Goldhaber \& Clarkson, 2011; Center on Media and Human Development, 2014). Caso não existissem tecnologias é possível concluir que: (i) a comunicação e interação entre o/a adolescente e a família manter-se-iam iguais, (ii) poderiam melhorar prevendo-se mais momentos de comunicação com a família, particularmente com os pais.

As conclusões da presente investigação permitem-nos reconhecer o poder das tecnologias na interação entre mães e filhos/as adolescentes, pela capacidade que estas têm de provocar alterações (positivas ou negativas). Permitem-nos, também destacar a importância e o valor da comunicação no seio da família, bem como a necessidade de se ajudar os adolescentes a encontrar um justo equilíbrio entre o tempo despendido com tecnologias e a comunicação com a família.

\section{Referências}

Abreu, C. (2012). La Teoría de los Grupos de Referencia.

Ágora - Papeles de Filosofia, 31(2), 287-309.

Alarcão, M. (2006). (Des) Equilíbrios Familiares. (3 ${ }^{\text {a }}$

Ed.), Coimbra: Quarteto Editora.

Beja, M. \& Franco, M. (2013). O Adolescente e a

Família: Autonomia ou Autonomias? In T. Medeiros. ( $1^{a}$ Ed.), Adolescência: Desafios e Riscos (pp. 269284). Ponta Delgada: Letras Lavadas.

Bovill, M. \& Livingstone, S. (2001). Bedroom Culture

and the Privatization of Media Use. In S. Livingstone and M. Bovill. (Eds.) Children and Their Changing Media Environment: a European Comparative Study (pp.179-200).

Center on Media and Human Development. (2014).

Revised Parenting in the Age of Digital Technology - A National Service. Northwestern University.

Church, K., Weight, J., Berry, M. \& MacDonald, H.

(2010). At Home with Media Technology. Home Cultures, 7(3), 263-286.

De Goede, I., Branje, S. \& Meeus, W. (2009).

Development Changes in Adolescent's Perceptions of Relationships with Their Parents. Journal of Youth Adolescence, 38, 75-88.

Fonseca, H. (2012). Compreender os Adolescentes: Um

Desafio para Pais e Educadores. Lisboa: Editorial Presença.

Kirchler, E., Palmonari, A., \& Pombeni, M. L. (1993).

Developmental Tasks and Adolescents' Relationships with Their Peers and Their Family. In S. Jackson \& H. Rodriguez-Tomé (Eds.), Adolescence and Its Social Worlds (pp. 145-167). Reino Unido: Lawrence Erlbaum Associates.

Hameededdin, N. (2010). New Communication

Technologies and Social Change: Cell Phone Effects on the Saudi Family. (Dissertação de Mestrado). Universidade Americana. Paraguai.

Lanigan, J., Bold, M. \& Chenoweth, L. (2009).

Computers in the Family Context: Perceived Impact on Family Time and Relationships. Family Science Review, 14, 17-32.

Livingstone, S. (2006). From Family Television to

Bedroom Culture: Young People's Media at Home. In. E. Devereux (Ed.), Media Studies: Key Issues and Debates (pp. 302-321). Londres: Sage Publications.

McGrath, S. (2012). The impact of new media

technologies on social interaction in the household. Electronic Culture and Social Change.

Mesch, G. (2006). Family Relations and the Internet:

Exploring a Family Boundaries Approach. Journal of Family Communication, 6(2), 199-138.

Mieczakowski, A., Goldhaber, T. \& Clarkson, J. (2011).

Culture, Communication and Change: Summary of an Investigation of the Use and Impact of Modern Media and Technology in our Lives. Engineering Design Centre, Universidade de Cambridge. 
Moraes, R. (1999). Análise de Conteúdo. Revista

Educação, 22(37), 7-32.

Morrison, M. \& Krugman, D. (2001). A Look at Mass

and Computer Mediated Technologies: Understanding the Roles of Television and Computers in the Home. Journal of Broadcasting \& Electronic Media, 45(1), 135-161.

Mota, C. \& Rocha, M. (2012). Adolescência e Jovem

Adultícia: Crescimento Pessoal, SeparaçãoIndividuação e o Jogo das Relações. Psicologia: Teoria e Pesquisa, 28(3), 357-366.

Neuenschwander, M. (2002). Desenvolvimento e

Identidade na Adolescência. Coimbra: Livraria Almedina.

Prados, M., Vicent, P. \& Esteban, S. (2014). La

Comunicacíon en la família a través de las TIC. Percepción de los adolescentes. Puls, 37, 35-38.

Quintas, S. (1998). Familia y Medios de Comunicación.

Comunicar, 10, 21-26.

Redmont, D. (2010). The Effect of Video Games on

Family Communication and Interaction. (Dissertação de Mestrado) Iowa State University. Iowa.

Rideout, V., Foehr, U. \& Roberts, D. (2010). Generation M2: Media in the Lives of 8-to 18-Year-Olds: A Kaiser Family Foundation Study. Menlo Park, California: Henry J. Kaiser Family Foundation.

Rodríguez, J. \& López, B. (1999). La familia y el adolescente. Revista Medica del Hospital General de México, 62(3), 191-197.

Saxbe, D., Graesh, A. \& Alvik, M. (2011). Television as a Social or Solo Activity: Understanding Families' Everyday Television Viewing Patterns. Communication Research Reports, 28(2), 180-189.

Subrahmanyam, K., Reich, S., Waetcher, N. \& Espinoza,

G. (2008). Online and Offline Social Networks: Use of Social Networking Sites by Emerging Adults. Journal of Applied Developmental Psychology, 29, 420-433.

Villegas, A. (2013). The influence of technology on

family Dynamics. Proceedings of the New York State Communication Association, 10, 1-17.

Wagner, A., Falcke, D., Silveira, L. \& Mosmann, C.

(2002). A comunicação em famílias com filhos adolescentes. Psicologia em Estudo, 7(1), 75-80. 